\title{
Muzzle Velocity Error Estimation and System Accuracy Prediction
}

\author{
[Gun In Kim, Hwan Il Kang, Hyun Soo Kim]
}

\begin{abstract}
To predict the system accuracy, we should calculate the measure of the muzzle velocity error and elevation difference according to $1 \mathrm{~m} / \mathrm{s}$ change of muzzle velocity. The magnitude of the muzzle velocity may be obtained by weighed sum of three quantities: the percentage errors of projectile weight, propellant weight and the volume of the chamber. The elevation difference according to $1 \mathrm{~m} / \mathrm{s}$ change of muzzle velocity can be obtained from the multivariable function. The inputs of the multivariable function are: the distance that the bullet falls due to gravity, the distance from the launch position to the target, muzzle velocity, tangent angle from the launch position to the target. The error on the target due to muzzle velocity error can be a product of two quantities: the measure of the muzzle velocity error and elevation difference according to $1 \mathrm{~m} / \mathrm{s}$ change of muzzle velocity. To predict the system accuracy, we use the accuracy function having the standard deviation and then we may obtain the system accuracy.
\end{abstract}

Keywords - system accuracy, muzzle velocity, target, accuracy function, projectile, propellant.

\section{Introduction}

To obtain system accuracy, we study error identification, error estimation, and the prediction of system accuracy [1]. We choose the muzzle velocity error as the most important error identification. For error estimation, we consider the magnitude of the muzzle velocity error and the error on the target due to the muzzle velocity error. The prediction of the system accuracy is obtained by using the normal distribution of the bullets on the target. In Section II, we present the known results for the error estimation and the relationship between muzzle velocity error and the target $[2,3]$. In Section III, we present our main results for the error estimation on the target and the prediction of system accuracy. Conclusions will be followed in Section IV.

Hwan Il Kang and Hyun Soo Kim

Dept. of Information \& Communication Engineering, Myongji University Myongj-ro, Cheoin-gu, Yongin, Republic of Korea

Gun In Kim

Dept. of Acquisition, Konkuk University

120, Heung-dong-ro, Gwang-jin-gu, Seoul, Republic of Korea

\section{Known Facts}

Before you describe the main useful results, main known facts are given in this section [2,3].

\section{A. Magnitude of Muzzle Velocity Error}

The Le Duc equation is expressible as

$$
v=\frac{a x}{(b+x)}
$$

where $a$ is a muzzle velocity when the barrel is assumed to be infinite and $b$ is a twice of the length at which the pressure inside the chamber volume is maximum. Specifically we may describe $a$ and $b$ as follows:

$$
a=J\left(W_{c} / W_{p}\right)^{1 / 2} \Delta^{n}
$$

$$
b=\beta\left(1-\frac{\Delta}{\delta}\right)\left(\frac{W_{c}}{W_{p}}\right)^{2 / 3} \Delta^{-2 / 3}
$$

where $J, W_{c}$, and $W_{p}$ are constant related to propellant, propellant weight, projectile weight, respectively. The notations, $\beta$ and $\delta$ are a constant related to distance and specific gravity of propellant, respectively and

$$
\Delta=\frac{W_{c} / V_{c h}}{W_{H_{2}} \mathrm{O} / V_{c h}}
$$

where $V_{c h}$, and $W_{\mathrm{H}_{2} \mathrm{O}}$ are a chamber volume and weight of water $\mathrm{H}_{2} \mathrm{O}$ inside the chamber volume. The muzzle velocity is given by

$$
V_{m}=\frac{a L}{(b+L)}
$$

where $L$ and $W_{\mathrm{H}_{2} \mathrm{O}}$ is a length of the barrel. Taking differentiation of the natural logarithm of equation (5), we obtain 
Proc. of the Sixth International Conference on Advances in Mechanical and Robotics Engineering - AMRE 2017.

Copyright (C) Institute of Research Engineers and Doctors. All rights reserved.

ISBN: 978-1-63248-140-5 doi: 10.15224/ 978-1-63248-140-5-39

$$
\frac{d V_{m}}{V_{m}}=\frac{d a}{a}+\left(1-\left(\frac{b}{b+L}\right)\right) \frac{d L}{L}-\frac{d b}{b+L}
$$

Inserting modification of equations (2) and (3) into (6), we obtain

$$
\begin{array}{r}
\frac{\mathrm{dV}_{\mathrm{m}}}{\mathrm{V}_{\mathrm{m}}}=\left[\frac{1}{2}+\mathrm{n}+\left(\frac{\mathrm{b}}{\mathrm{b}+\mathrm{L}}\right)\left(\frac{\Delta / \delta}{1-\Delta / \delta}\right)\right] \frac{\mathrm{dW}_{\mathrm{c}}}{\mathrm{W}_{\mathrm{c}}}- \\
{\left[\frac{1}{2}-\frac{2}{3}\left(\frac{\mathrm{b}}{\mathrm{b}+\mathrm{L}}\right)\right] \frac{\mathrm{d} \mathrm{W}_{\mathrm{p}}}{\mathrm{W}_{\mathrm{p}}}-\left[\mathrm{n}+\left(\frac{\mathrm{b}}{\mathrm{b}+\mathrm{L}}\right)\left(\frac{2}{3}+\frac{\Delta / \delta}{1-\Delta / \delta}\right)\right] \frac{\mathrm{dV}_{\mathrm{ch}}}{\mathrm{V}_{\mathrm{ch}}}}
\end{array}
$$

which is described in the book [2].

\section{B. Some Known Facts for Relationship Muzzle Velocity and Target}

All the equations are given by the paper [3]. The bullet equations are given by

$$
\begin{gathered}
\ddot{x}(t)=H(t) \dot{x}(t) \\
\ddot{z}(t)=H(t) \dot{z}(t) \\
\ddot{y}(t)=H(t) \dot{y}(t)-g \\
H(t)=-\left(\frac{i s}{2 w}\right) \rho_{g} \exp \left(-f_{a} y\right) C_{d} v(t)
\end{gathered}
$$

where the $x$ axis is the range direction of the bullet, the $z$ axis is the cross range direction and the $y$ axis is the opposite direction against the gravity direction. The notations $i, S, w, \rho_{g}, f_{a}, C_{d}, v(t)$ are forming constant, sectional area of the bullet, mass of the projectile(bullet), air density, altitude constant, drag coefficient and the velocity of the bullet, respectively. From the equation

$$
v^{2}=\dot{x}^{2}+\dot{y}^{2}+\dot{z}^{2}
$$

Differentiation of (12) gives us

$$
\dot{v}=\frac{\dot{x}}{v} \ddot{x}+\frac{\dot{y}}{v} \ddot{y}+\frac{\dot{z}}{v} \ddot{z}
$$

The equations (11),(12) and (13) leads to

$$
\dot{v}=H(t) v(t)-\frac{g}{v} \dot{y} \approx H(t) v(t) \text {. }
$$

Assume that the distance from the launch position to the target is $D$, the flight time of the projectile is $T_{f}$, the additioal superelevation angle $\alpha$ which means that the angle $\alpha$ allows compensation for the drop due to the gravity as shown in Fig. 1.

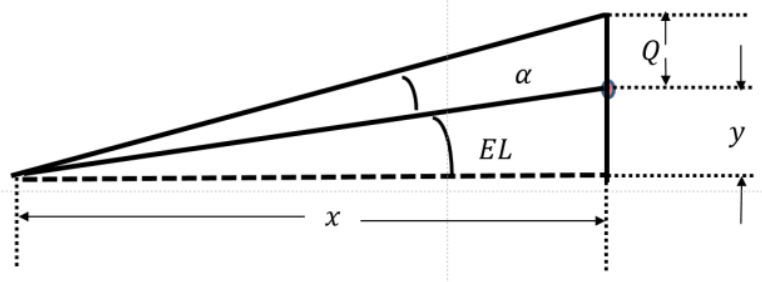

Figure 1. Superelevation and Q

We may obtain the equations:

$$
\begin{array}{r}
T_{f}=\frac{D}{B-\beta D} \\
Q=\frac{1}{2} g T_{f}^{2}\left(1-\frac{2 \beta}{3 B} D\right) \\
\alpha=\tan ^{-1} \frac{D \sin E L+Q}{D \cos E L}-E L
\end{array}
$$

where $B$ is a muzzle velocity,

$$
\begin{array}{r}
\beta=\frac{C_{1} \sqrt{B}}{2} \\
C_{1}=\left(\frac{i s}{2 w}\right) \rho_{g} \exp \left(-f_{a} \bar{y}\right) A_{1} \sqrt{v_{s}} \\
\rho_{g} v_{s}=\rho_{s} \frac{P}{273+T}
\end{array}
$$

where $\rho_{s}$ is the air density at the standard time, $P$ is the air pressure at the launch position, $T$ is atmospheric Celcius temperature, $v_{s}$ is the sound of the sound. Equations (15) and (16) leads to

$$
Q=\frac{g D^{2}}{2(B-\beta D)^{2}}\left(1-\frac{2 \beta}{3 B} D\right)
$$
are

From the above equations, the following first derivatives obtained:

$$
\begin{array}{r}
\frac{d \alpha}{d B}=\frac{\frac{d Q}{d B} D \cos E L}{(D+Q \sin E L)^{2}} \\
\frac{d \alpha}{d E L}=\frac{-Q(Q+R \sin E L)}{(D+Q \sin E L)^{2}}
\end{array}
$$


Proc. of the Sixth International Conference on Advances in Mechanical and Robotics Engineering - AMRE 2017.

Copyright (C) Institute of Research Engineers and Doctors. All rights reserved.

ISBN: 978-1-63248-140-5 doi: 10.15224/ 978-1-63248-140-5-39

$$
\frac{d Q}{d B}=\frac{g D^{2}\left(\beta B D-\beta^{2} D^{2}-3 B^{2}\right)}{3 B^{2}(B-\beta D)^{3}}
$$

\section{Main Results}

We are in a position to present main results. The equation connecting the muzzle velocity error with the target is given by

$$
\frac{d(\alpha+E L)}{d B}=\frac{d \alpha}{d B}+\frac{d E L}{d \alpha} \frac{d \alpha}{d B}=\frac{d \alpha}{d B}\left(1+\frac{d E L}{d \alpha}\right)
$$

Using the equation (23), the equation (24) goes to

$$
\frac{d(\alpha+E L)}{d B}=\frac{\frac{d Q}{d B} D \cos (E L)}{(D+Q \sin (E L))^{2}}\left(1-\frac{(D+Q \sin (E L))^{2}}{Q(Q+D \sin (E L))}\right)
$$

The muzzle velocity error is rewritten from the equation (7) as

$$
\begin{array}{r}
d V_{m}=V_{m}\left(\left[\frac{1}{2}+n+\left(\frac{b}{b+L}\right)\left(\frac{\Delta / \delta}{1-\Delta / \delta}\right)\right] \frac{d W_{c}}{W_{c}}-\right. \\
\left.\left[\frac{1}{2}-\frac{2}{3}\left(\frac{b}{b+L}\right)\right] \frac{d W_{p}}{W_{p}}-\left[n+\left(\frac{b}{b+L}\right)\left(\frac{2}{3}+\frac{\Delta / \delta}{1-\Delta / \delta}\right)\right] \frac{d V_{c h}}{V_{c h}}\right)
\end{array}
$$

Assuming that the error of the muzzle velocity is Gaussian, then the standard deviation of the muzzle velocity error is

$$
\begin{array}{r}
\sigma_{B}=V_{m}\left(\left[\frac{1}{2}+n+\left(\frac{b}{b+L}\right)\left(\frac{\Delta / \delta}{1-\Delta / \delta}\right)\right] \frac{d W_{c}}{W_{c}}-\right. \\
\left.\left[\frac{1}{2}-\frac{2}{3}\left(\frac{b}{b+L}\right)\right] \frac{d W_{p}}{W_{p}}-\left[n+\left(\frac{b}{b+L}\right)\left(\frac{2}{3}+\frac{\Delta / \delta}{1-\Delta / \delta}\right)\right] \frac{d V_{c h}}{V_{c h}}\right)
\end{array}
$$

The standard deviation on the target is given by

$$
\sigma_{B T}=\sigma_{B} \frac{d(\alpha+E L)}{d B}
$$

The system accuracy is given by

$$
p(h)=\frac{1}{\sqrt{2 \pi} \sigma_{B T}} \int_{-\frac{h}{2}}^{\frac{h}{2}} \exp \left(-\frac{y^{2}}{2 \sigma_{B T}^{2}}\right) d y
$$

The muzzle velocity error may be a weighted sum of three quantities: percentage error of projectile weight, propellant weight and the chamber volume. If we assume that the error of the muzzle velocity error is Gaussian, we may obtain the standard deviation of the muzzle velocity error. In addition we obtain the error on the target due to the unit quantity change in muzzle velocity error. Multiplying the standard deviation of the error by the error on the target due to the unit quantity change in muzzle velocity error, we obtain the standard deviation on the target, By system accuracy prediction equation, we obtain the system accuracy due to muzzle velocity error. This approach will be a good resources for theoretical prediction of system accuracy.

\section{Acknowledgment}

This work was supported by the Research program (the specialized research center for future ground system analysis (Contract No. 311165-911074201) funded by the agency for defense development in the republic of Korea.

\section{References}

[1] H. I. Kang, G. I. Kim, and H. S. Kim, "A study on prediction of probability of hit for an anti-aircraft artillery," Indian Journal of Science and Technology, vol. 9, no. 1, Dec., 2016.

[2] Heung Joo Lee, "Gun and Ballistics," Cheongmun-gak, Seoul, Republic Korea, 2008.

[3] Jaehun Kim, Euiwhan Kim, Jeongyeob Lee, Gunkook Kim, "A derivation of sensitivity equations of the error components to anlyze performance in the direct fire control system," KIPS Transactions of computer and communication systems, vol. 4 , no. 12, pp. 399-408, April, 2015.

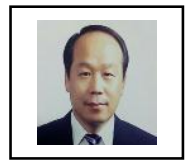

GUN IN KIM was born in Seoul, South Korea and graduated from the Korea Military Academy, Yonsei University and University of Washington in USA where he studied mechanical engineering and obtained his BS, MS and Ph. D. degrees, respectively. He has been a professor at dept. of Defense Acquisition In Konkuk University, South Korea since 2015. His research interest includes modelling \& simulation, military sciences and control applications.

\section{Conclusions}

